\title{
CHOOSING EFFECTS OF PHYSICAL CARRIER SENSING IN WIRELESS AD HOC NETWORKS
}

\author{
Shankar . $T^{1}$, Dr.Shanmugavel . $S^{2}$, Sandeep . $V^{3}$ \\ ${ }^{1,2,3}$ Dept. Electronics and Comm.Engg, CEG, Anna University-Chennai. \\ E-mail : 'tshankar77@gmail.com
}

\begin{abstract}
IEEE 802.11 MAC having two methods for medium reservation. 1) virtual carrier sensing and 2) physical carrier sensing. VCS having many drawbacks in wireless ad hoc networks. PCS mechanism can replace the virtual carrier sensing in most practical applications. But choice of PCS threshold plays vital role in ad hoc networks. Choice of PCS threshold is tradeoff between the throughput and spatial reuse. In this paper we present practical experiments for to see the PCS threshold effects on network throughput.
\end{abstract}

Keywords: throughput; physical carrier sensing; MAC; ad hoc network; IEEE 802.11; simulation

\section{INTRODUCTION}

Nowadays ad hoc networks are providing broadband connectivity to the backbone networks for Internet for mobile clients such as campus, office and home must exploit the limited system bandwidth available via spatial reuse to enhance aggregate 1-hop throughput. However, enhancing spatial reuse in such dense ad hoc networks depends on various factors [1]: the type of radio, signal propagation environment and network topology. In particular, the random topology of an ad hoc network has a significant impact on interference management and can cause large local variability in achievable spatial reuse.

In IEEE 802.11, Distributed Coordinating Function (DCF) [2-4] or CSMA/CA uses carrier sensing to determine if the shared medium is available before transmitting. Two types of carrier sensing are supported by DCF: mandatory physical carrier sensing [2] monitors RF energy level in the medium and optional virtual carrier sensing [3] using RTS (request to send) and CTS (clear to send) to preserve the medium.

Before going into the concepts of Physical Carrier Sensing let us see the braw backs of Virtual Carrier Sensing in section II. Interference mitigation via PCS is explained in section III. And last section deals with simulation results how Physical Carrier Sensing threshold (range) affects the network throughput.

\section{NECESSITATE OF PHYSICAL CARRIER SENSING}

In wireless networks mobility leads to interference with other nodes. Interference mainly happens with hidden terminal problems. So to resolve the hidden terminal problem [4] becomes one of the major design considerations of 802.11 MAC protocols. IEEE 802.11 DCF is the most popular MAC protocol used in both wireless LAN and ad-hoc networks. Its RTS/CTS handshake is mainly designed for such a purpose. However, it has an underlying assumption that all hidden nodes are within the transmission range of receivers. In this paper we can see such an assumption man not hold when the transmitter-receiver distance exceeds a certain value. In Fig 1 shows this distance effect on performance.

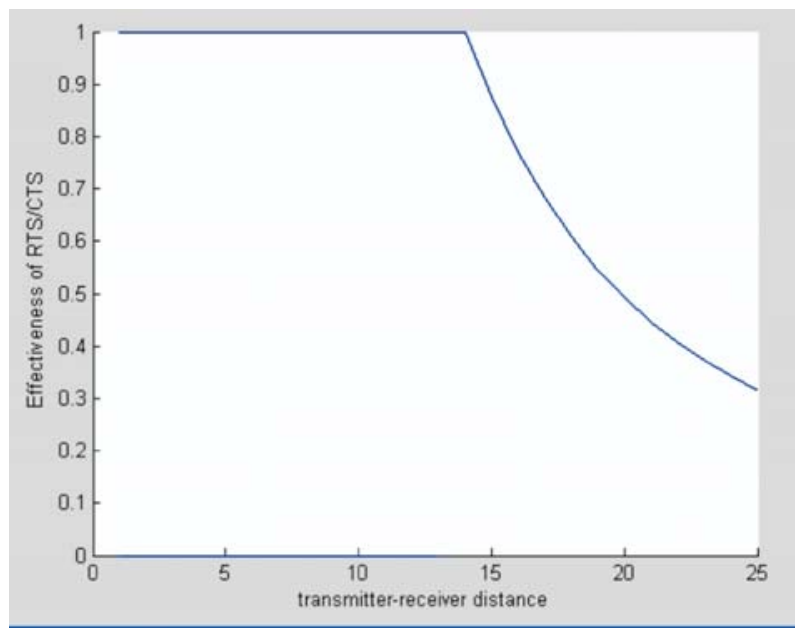

Fig. 1. Effectiveness of Virtual Carrier Sensing

We consider a 1-D chain network for simulation results. In this chain networks all nodes are placed in one by one and distance between the nodes is $200 \mathrm{~m}$. When distance between the transmitter-receiver is $200 \mathrm{~m}$ which is large value then the interference range is $356 \mathrm{~m}$. Basically in first case throughput is nearly $600 \mathrm{Mbps}$ very less because of large distance between the nodes. Throughput is decreased because of hidden terminal problem. 


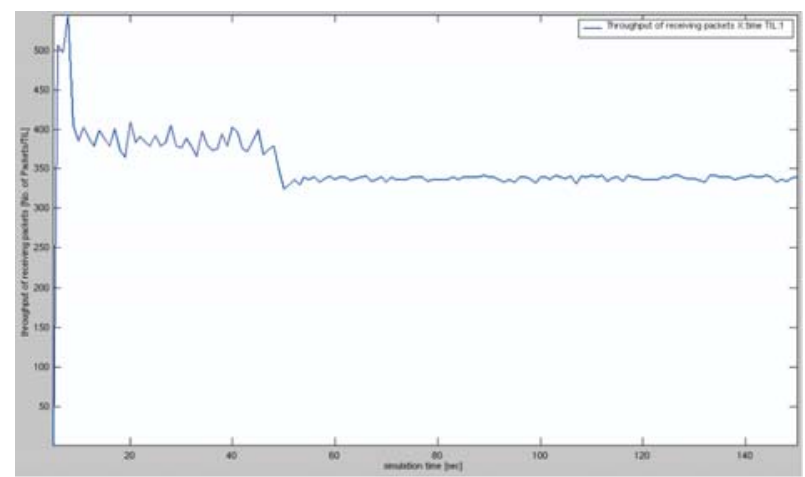

\section{i g 2. Ef fect ivenesso f I mgd i $\$$ anceo nRTS/ $\sigma S$}

In second case distance between the transmitter and receiver is $150 \mathrm{~m}$. Then the interference range is occupied up to $267 \mathrm{~m}$. In this situation distance between the transmitter-receiver is very less so throughput very high nearly $1200 \mathrm{Mbps}$ from Fig. 3 .

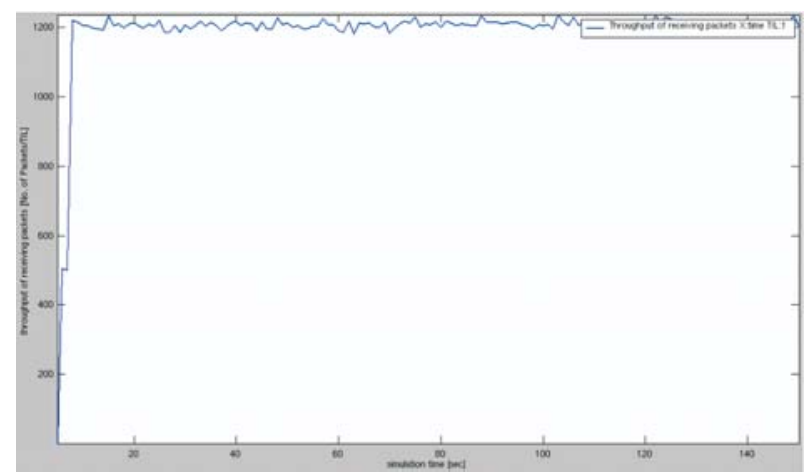

Fig. 3. effectiveness of small distance on RTS/CTS

We can conclude when the transmitter-receiver distance is less I can get maximum throughput because of hidden terminals are very less. Otherwise in second case throughput is minimum because hidden terminals are very high. Practical applications such assumption can hold properly not only this reason RTS/CTS handshake won't give maximum spatial reuse. Physical Carrier Sensing can eliminate all this drawbacks.

\section{INTERFERENCE MITIGATION VIA PCS}

In this section, we discuss the properties of radio communication that determine the effectiveness of carrier sensing and point out several shortcomings of the carrier sensing techniques commonly employed in 802.11 MAC [5].

\section{A. Communication model}

Pathloss models are commonly used to describe the radio propagation property in wireless networks [6]. A typical pathloss model expresses the average signal strength at the receiver as a function of the T-R (transmitter-receiver) separation distance, d, i.e.

$$
P_{r x}(d)=\bar{P}_{r x}\left(\frac{\bar{d}}{d}\right)^{\gamma}
$$

Where $\gamma$ is the pathloss exponent that characterizes how quickly a signal fades in the particular network environment. $P_{r}(d)$ denotes the signal strength at a receiver at distance $d$ away. Finally, $\bar{P}_{r}$ is the reference receiving signal strength as measured at the reference distance $\bar{d}$ (usually 1 meter). The aggregate energy detected by a receiver consists of signal (from intended transmitter), interference (from unwanted transmitter(s)) and noise. In ad hoc networks, a receiver can receive a packet with high probability of success only if the receiving strength of the intended signal is greater than a threshold (denoted by $P_{R}$ ), and the signal-noise-interference ratio $\left(S N I R\right.$ ) is above a threshold (denoted by $5_{0}$ ).

$$
\begin{gathered}
P_{r x}(d) \geq P_{R} \\
\frac{P_{r x}(d)}{P_{N}+\sum P_{r x}\left(d_{i}\right)} \geq S_{0}
\end{gathered}
$$

Where $P_{N}$ is the strength of the ambient noise, and $P_{r x}(d)$ denotes the signal strength from interference sources i at distance. In most cases, the noise level is negligible compared to either the signal and interference.

\section{B. Interference model}

According to above equation, a successful reception depends on the receiving power as well as the runtime SNIR. Hence there does not exist a static transmission range irrespective to the network environment and runtime traffic pattern. Nonetheless, when there is no interference, the noise will determine the maximum distance for reception. Let transmission range denote that distance. For each network, we can assume such transmission range exists. Similarly, in the rest of the thesis, let us use simplistic terms such as interference range, carrier sensing range, etc. to facilitate analysis.

Before going in detail we must know basic definitions like what is meant by transmission range, interference range and carrier sensing range. Figure 4 shows a segment in a typical ad hoc network with a reference transmission from $T X$ to $R X$ and four other nodes $(A, B, C$, and $E)$. The same transmission power is used by every node in the network. We define the following notations:

D: T-R separation distance,

$\mathrm{R}$ : Transmission range, given by 
$R=\bar{d}\left(\frac{\bar{P}_{r x}}{P_{R}}\right)^{\frac{1}{\gamma}}$

I: Interference range - an energy level equivalent to a transmitter within that distance of the receiver will disrupt the reception, given by

$I=D\left[\frac{1}{S_{0}}-\left(\frac{D}{d}\right)^{\gamma} \frac{P_{N}}{P_{r x}}\right]^{\frac{1}{\gamma}}$

With negligible noise, then above equation becomes

$$
I=S_{0}^{\frac{1}{\gamma}} D>D
$$

$\mathrm{X}$ : Physical carrier sensing range - a transmitter will deem channel busy if it senses an energy level equivalent to a transmitter within that range, given by

$X=\bar{d}\left(\frac{\bar{P}_{r x}}{P_{C}}\right)^{\frac{1}{\gamma}}$

Where $P_{c}$ denotes the carrier sensing threshold.

With Physical Carrier Sensing (PCS), a station compares the energy level against the sensing threshold $\left(P_{c}\right)$, and starts transmission only when the reading is below $P_{c}$.

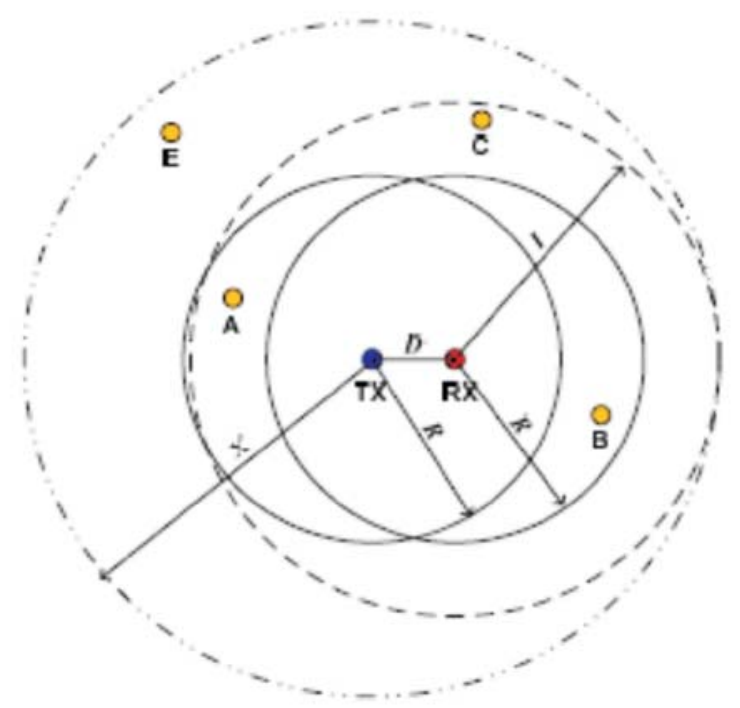

Fig. 4. Communication in a wireless ad hoc network

In Fig. 4, all interfering nodes, including $C$, can be reduce enlarging the sensing range $(X)$ to cover the entire interference area, i.e. $X \geq D+1$. Therefore, any node within the sensing range of $\mathrm{TX}$ will be able to detect the on-going transmission between $\mathrm{TX}$ and $\mathrm{RX}$, therefore refrain from transmitting to avoid generating interference. From I and $X$ we can

write $\bar{d}\left(\frac{\bar{P}_{r x}}{P_{C}}\right)^{\frac{1}{\gamma}} \geq D\left(1+S_{0}^{\frac{1}{\gamma}}\right)$

That leads to

$$
P_{C S_{-} t} \leq \frac{1}{\left(1+S_{0}^{\frac{1}{\gamma}}\right)^{\gamma}}
$$

The PCS threshold $P_{c}$ plays a key role to coordinate simultaneous transmissions for optimal spatial reuse and throughput. PCS generally is more robust than VCS since it does not require packets to be received correctly.

\section{IV.SIMULATION RESULTS}

In this section, we investigate the performance of PCS threshold on throughput and spatial reuse. We use ns-2 [10] as the network simulator. The choice of PCS threshold is trade-off between the probability of collision and spatial reuse. Higher values of physical carrier sensing threshold leads to low throughput or high probability of collision and one high spatial reuse. Lower values of physical carrier sensing threshold leads to high throughput or low probability of collisions and low spatial reuse. In both cases having advantages and disadvantages so we have take one optimal threshold value which will optimize this disadvantage. To demonstrate the effects the PCS threshold on probability of collisions and throughput, we did a simple experiment using NS-2.27. The topology of experiment is demonstrated in Fig. 5.

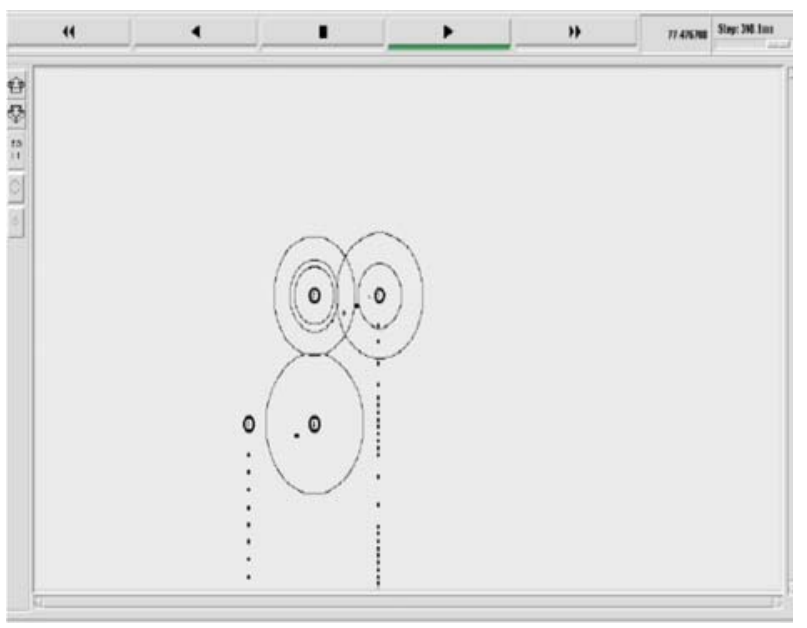

Fig. 5. Scenario for investigation of PCS threshold on throughput 
The distance from node 1 to node 2 and node 3 to node 4 is fixed as $300 \mathrm{~m}$. Transmission range of the wireless radio is $250 \mathrm{~m}$ with channel bandwidth as $2 \mathrm{Mbps}$. We vary the vertical distance between node 3,4 and node 1, 2 to check the influence of PCS threshold. Two CBR sessions based on UDP are involved with directions from node 1 to node 2 and node 4 to node 3 correspondingly. Since the CBR is constant rate traffic without retransmissions, it is possible that the two flows may synchronize to each other rendering the results not general enough. The packet rates of two CBR flows are set to $800 \mathrm{Kbps}$ with packet size 1024 bytes (thus 100 packets per second). The packet rate of CBR is selected as to utilize the full bandwidth when the two flows share the channel (e.g. the available channel bandwidth to each flow is $1.7 \mathrm{Mbps} / 2=850 \mathrm{Kbps})$. It is interesting to notice that when the PCS threshold is equal to transmission range which is very low value and in second case PCS threshold is large value greater than the transmission range which is very big value.

Consider first case physical carrier sensing is equal to transmission range $(250 \mathrm{~m}=250 \mathrm{~m})$. we can see the throughput in Fig. 6 is very high value nearly 1400Mbps.

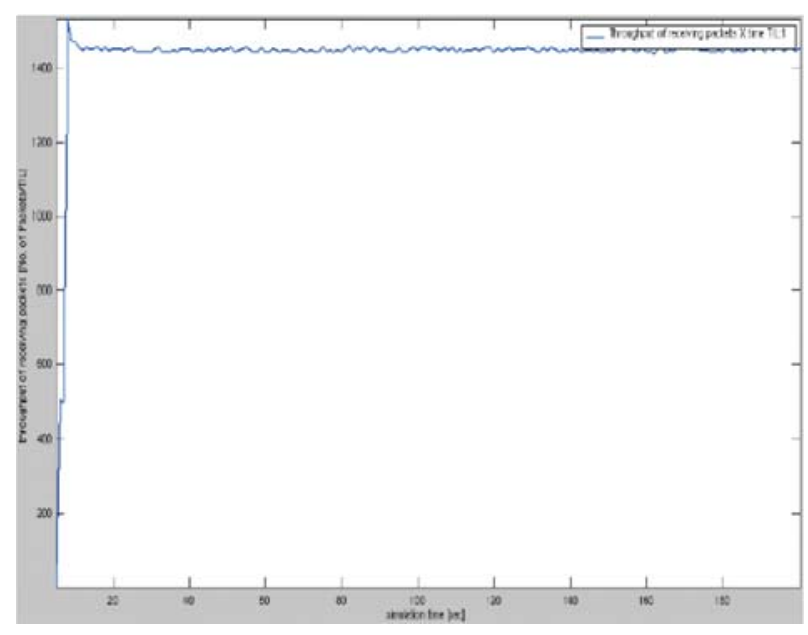

Fig. 6. Effects of lower PCS threshold value on network throughput

This because of there is no hidden terminal in transmission range of transmitter (node 0 ). In this case very less chance of probability of collision because of node 2 and 3 are out range. These two nodes are not disturbing the transmission between nodes 0 and 1. But one drawback is spatial reuse is very low.

In second case we consider physical carrier sensing $(500 \mathrm{~m})$ is equal to double of the transmission range $(250 \mathrm{~m})$. In this case we can observe more hidden terminals in the transmission range of transmitter (node 0 ) from figure 5 .

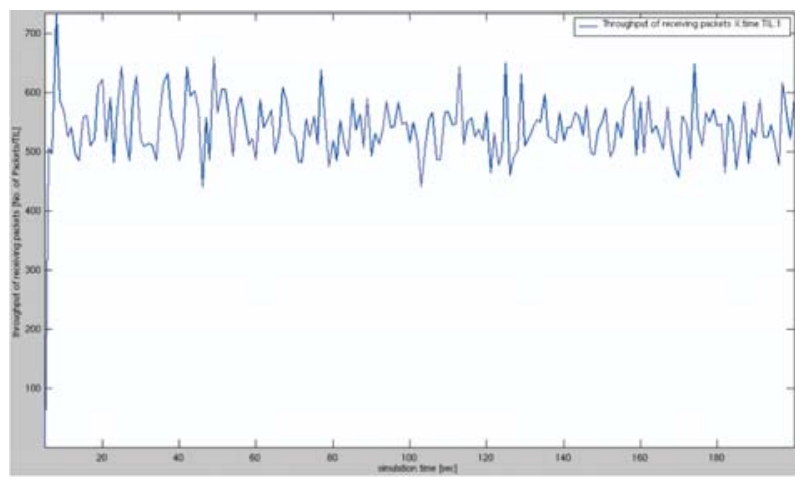

Fig. 7. Effects of higher PCS threshold value on network throughput

So node 2 and node 3 are disturbing present on going transmission from node 0 to node 1.from the Fig 7 we can observe total network throughput is $700 \mathrm{Mbps}$. From the above two cases throughput (or probability of collisions) is less in very less in second case compared to first case this because of hidden terminal presented in environmental network.

\section{CONCLUSIONS}

From the above work we can say choosing Physical Carrier Sensing (PCS) range plays an important role in wireless networks. It is not a very large value which leads to less throughput (High probability of collisions) Otherwise it is not a very less value which leads to large throughput (less probability of collisions).so finally Physical carrier sensing with the optimal sensing threshold is effective at leveraging throughput in multi-hop ad-hoc networks. Such improvement does not require the use of virtual carrier sensing.

\section{REFERENCES}

[1] X. Guo, S. Roy, W. Steven Conner, "Spatial Reuse in Wireless Ad-Hoc Networks," VTC2003.

[2] J. Zhu, X. Guo, L. Lily Yang, W. Steven Conner, S. Roy, Mousumi M. Hazra, "Adapting physical carrier sensing to maximize spatial reuse in 802.11 mesh networks," Wireless Communications and Mobile Computing Volume 4, Issue 8, p 933-946, December 2004.

[3] K. Xu, M. Gerla, S. Bae, "How effective is the IEEE 802.11 RTS/CTS handshake in ad hoc networks?" GLOBECOM '02, Nov. 2002.

[4] F. A. Tobagi and L. Kleinrock, "Packet Switching in Radio Channels: Part II - The Hidden Terminal Problem in Carrier Sense Multiple Access and the Busy Tone Solution," vol. COM-23, no. 12, pp. 1417-1433, 1975. 
[5] J. Zhu, X. Guo, L. L. Yang, and W. S. Conner, "Leveraging Spatial Reuse in 802.11 Mesh Networks with Enhanced Physical Carrier Sensing," ICC '04, June 2004.

[6] Theodore S. Rappaport, Wireless Communications, Principles and Practices, 2nd Ed. Prentice Hall, 2002. ISBN 0-13-042232-0.

[7] IEEE P802.11. Standard for Wireless LAN Medium Access Control (MAC) and Physical Layer (PHY) Specifications, 1997.

[8] IEEE P802.11b. Supplement to Standard IEEE 802.11, Higher speed Physical Layer (PHY) extension in the $2.4 \mathrm{GHz}$ band, 1999.

[9] IEEE P802.11a. Supplement to Standard IEEE 802.11, High speed Physical Layer (PHY) extension in the $5 \mathrm{GHz}$ band, 1999.
[10] K. Fall and E. K. Varadhan, "Ns notes and documentation." [Online]. Available: http://www.isi.edu/nsnam/ns/

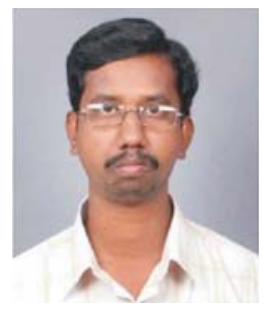

T. Shankar received the B.E. degree in Electronics and Communication Engineering from University of Madras, Tamil Nadu, India in 1999, and the M.E Applied Electronics from College of Engineering Guindy, Anna University Chennai, Tamil Nadu, India in 2005 and Ph.D doing in CEG, Anna university, Chennai, Tamil Nadu India. His research interests are in the area of mobile ad-hoc networks, software router design and systems security. Currently he is an Assistant professor in teaching field. He is a Life member in ISTE (Indian Society for Technical Education). 